\title{
COMMISSION 19: EARTH ROTATION (ROTATION DE LA TERRE)
}

$\begin{array}{ll}\text { President: } & \text { J.Vondrák } \\ \text { Vice-President: } & \text { D.D.McCarthy }\end{array}$

Organizing Committee:

$\begin{array}{llll}\text { F.Arias } & \text { N.Capitaine } & \text { V.Dehant } & \text { S.Dickman } \\ \text { S.Yang Fumin } & \text { S.Manabe } & \text { W.Melbourne } & \text { L.Morrison } \\ \text { D.Robertson } & \text { L.Rykhlova } & \text { V.Tarady } & \text { Ch.Veillet } \\ \text { P.Wilson } & \text { M.Feissel - IERS repr. } & \text { J.O.Dickey - IAG WG repr. } & \text { B.Kolaczek-past president }\end{array}$

\section{INTRODUCTION}

The work of Commission 19 in the period 1993.5-1996.5 was concentrated both on observational and theoretical aspects of Earth rotation, namely on the mutual interaction of the rotating non-rigid Earth with other Solar System bodies as well as with the atmosphere and fluids (oceans, underground water). The activities were closely tied with the work of the following bodies, supported or co-supported by the Commission:

- the International Earth Rotation Service (director of the Central Bureau: M. Feissel),

- the Commission 19 Working Group on Earth Rotation in the Hipparcos reference frame (chairperson: J. Vondrák),

- the intercommission Working Group on Astronomical Standards (chairperson: T. Fukushima),

- the intercommission Working Group on Reference Frames (chairperson: L. Morrison),

- the IAU/IUGG Working Group on the non-rigid Earth nutation theory (chairperson: V. Dehant), individual observatories and members of the Commission. The description of all these activities follows.

\section{REPORT OF THE INTERNATIONAL EARTH ROTATION SERVICE (IERS)}

\subsection{MISSIONS OF IERS}

The International Earth Rotation Service (IERS) is a member of the Federation of Astronomical and Geophysical Data Analysis Services (FAGS), created in 1988 by the IUGG and the IAU. It is in charge of providing basic reference frames to the international scientific community; it maintains a terrestrial and celestial reference frame, and monitors the Earth Orientation Parameters (EOP) which relate the two frames as a function of time.

The celestial reference system established by the IERS is in the process of being recommended by the IAU as the International Celestial Reference System (ICRS), replacing of the FK5 star catalogue. It is realized by the coordinates of extragalactic radio-sources included in the International Celestial Reference Frame (ICRF). IERS is in charge of its maintenance and of the link to other celestial reference frames. The Hipparcos catalogue and the Jet Propulsion Laboratory ephemerides are referred to the ICRS.

The terrestrial reference system established by the IERS is recommended by the IAG and the IUGG as the International Terrestrial Reference System (ITRS). It is realized by sets of station coordinates and velocities of sites included in the International Terrestrial Reference Frame (ITRF). IERS is in charge of its maintenance.

The Earth rotation and reference frame results compiled and distributed by IERS are accurate and precise at the level of $\pm 0.3 \mathrm{mas}$, corresponding $1.0 \pm 1 \mathrm{~cm}$ on the surface of the Earth.

The irregular fluctuations in the rotation of the Earth on sub-decadal time scales are driven mostly by changes in the angular momentum of the atmosphere. Thus, knowledge of the state of the atmosphere is fundamental to understanding signals in Earth orientation. Consequently the IERS extends its activity to the monitoring of the atmospheric winds and pressures, through linkage with meteorological agencies.

IERS is involved in international time service activity. It is in charge of deciding the time of introducing leap seconds in UTC (Coordinated Universal Time) that will keep it within \pm 0.9 seconds of UT1. 
The scientific and operational work and the evolution of the IERS are controlled by its Directing Board. The latter met seven times in 1994-1996 and organized three Workshops in Paris, the 1994 one being joint with the International GPS Service for Geodynamics (IGS), 70-80 scientists who are experts in the various fields of activity of IERS are appointed as Corresponding Members; they participate in the permanent evaluation of the service.

\subsection{OBSERVATIONS FOR IERS}

The study of the Earth's rotation and the maintenance of the ICRF and ITRF is based on the coordinated use of the modern observational techniques. The observing effort involves Very Long Baseline radio Interferometry (VLBI), Lunar Laser Ranging (LLR), Global Positioning System (GPS), Satellite Laser Ranging (SLR) and DORIS (Doppler Orbit determination and Radiopositioning Integrated on Satellite), all based on worldwide networks totalling over 300 stations in 1996. The close cooperation with the IGS, a FAGS service created in 1994 by the IUGG, is of special importance. While the IERS expertise in reference frames and Earth rotation is fully available to IGS, the permanent IGS orbit determination activity provides, as a by-product, daily monitoring of a part of Earth orientation information (polar motion and high frequency variations of universal time), while its regional positioning activity provides results of high consistency for the densification of, and access to, the ITRF. The atmospheric data related to Earth-orientation variations from four major meteorological centres are collected, validated and made available by the Sub-Bureau for Atmospheric Angular Momentum.

\subsection{STATUS OF IERS RESULTS IN 1996}

The current IERS results can be summarized as follows:

- Celestial frame: 610 extragalactic objects observed by VLBI, including 212 definition sources that materialize the ICRS. About $2 / 3$ of these objects have position accuracies smaller than 0.5 mas.

- Terrestrial frame: 240 sites with rms position uncertainties between 5 and $15 \mathrm{~mm}$ and velocity uncertainties of the order of $1 \mathrm{~mm} /$ year, determined by one or several of the five IERS techniques.

- Earth rotation: daily polar motion and UT1-UTC, correction to nutation and precession, all with an uncertainty of 0.2 mas.

- Accuracy of the tie of Earth rotation parameters with the celestial reference system $( \pm 0.03$ mas $)$ and the terrestrial reference system $( \pm 0.13$ mas at $1993.0 ; \pm 0.05$ mas/year $)$.

\subsubsection{Celestial References for Astrophysics and Geodynamics}

The ICRS (Arias et al. 1996) has its origin at the barycentre of the solar system. The directions of its axes are fixed with respect to the quasars to better than \pm 10 microarcseconds; they are aligned with those of the FK5 within the uncertainty of the latter ( \pm 80 mas). It is realized by estimates of the coordinates of a set of quasars in the ICRF.

A Working Group on Reference Frames (WGRF), with the participation of IERS, was appointed by the

$90^{\circ}$

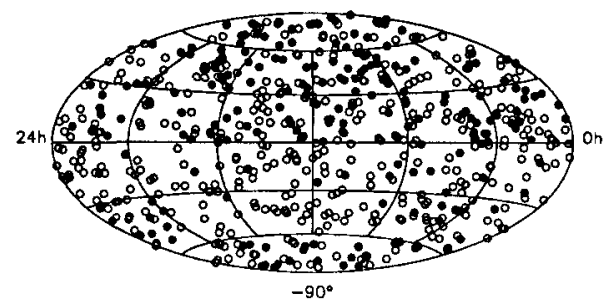

Figure 1. Sky distribution of the 610 sources in RSC(WGRF) $95 \mathrm{R}$ 01. Filled circles represent the 212 defining sources.

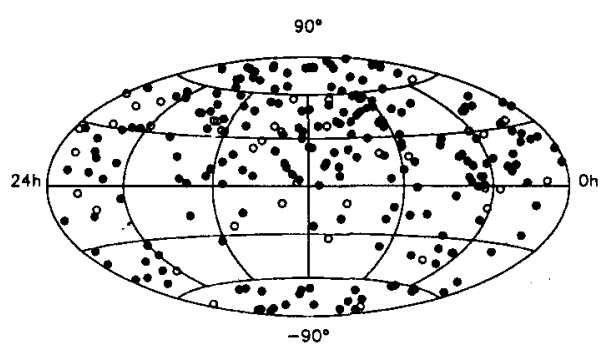

Figure 2. Sky distribution of the 253 sources in RSC(WGRF) $95 \mathrm{R} 02$. Filled circles represent the 195 defining-only sources, asterisks represent the 17 defining/link sources, circles represent the 41 link sources.

IAU to accomplish the task of constructing the ICRF. The WGRF elaborated a celestial reference frame that contains J2000.0 VLBI equatorial coordinates of 610 extragalactic radio sources, based on radio 
sources observed with dual frequency S/X Mark III VLBI up to July 1995. Efforts have been made in the derivation of source coordinates to minimize systematic inconsistencies coming from the modeling. The set of coordinates of the 610 objects in the ICRS is labelled RSC(WGRF) 95 R 01. Its sky distribution is shown in Fig. 1, and it includes the 58 extragalactic radio sources that served to link the Hipparcos stellar reference frame to the extragalactic reference frame.

A subset of defining objects was selected on the basis of number and time span of observations, detection of structure and apparent rate, and formal uncertainty of the derived coordinates. In order to provide radio source coordinates to link the Hipparcos reference frame to the ICRS, the WGRF issued a shorter version of the ICRF, RSC(WGRF) $95 \mathrm{R} 02$, that includes the 212 defining sources and the 58 Hipparcos link objects, 41 of which were not selected as definition sources; its sky distribution is plotted in Fig. 2. The histograms for its right ascension and declination uncertainties are shown in Fig. 3. The adoption of the ICRS and its materialization by the ICRF will be recommended to the IAU at its the next General Assembly (Kyoto, August 1997).

\subsubsection{Terrestrial References}

\section{for Geodesy, Geodynamics and Oceanography}

The ITRS origin is at the centre of mass of the whole Earth, including the oceans and the atmosphere. Its length unit is the metre (SI), defined in a local Earth frame in the meaning of a relativistic theory of gravitation. The orientation of its axes is consistent with that of the BIH System at 1984.0 within \pm 3 mas. Its time evolution in orientation is such that it has no residual rotation relative to the Earth's crust. The most recent realization of the ITRF is based on 240 sites, including over 60 observed by two or more techniques (VLBI, LLR, GPS, SLR, DORIS).

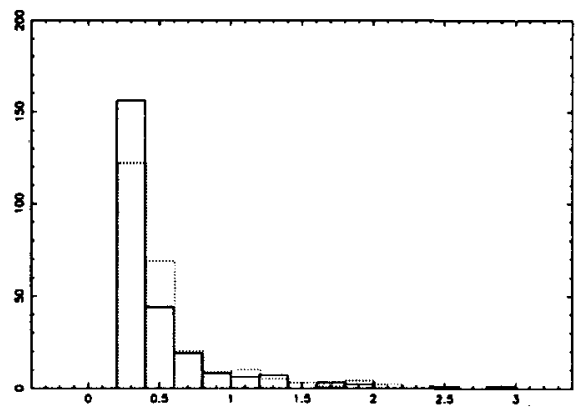

Figure 3. Distribution of formal uncertainties in right ascension (full line) and declination (dashed line) of the source position in RSC(WGRF) $95 \mathrm{R} 02$.

\subsubsection{Earth Rotation}

The sub-Bureau for Rapid Service and Predictions computes and distributes quick-look Earth orientation parameters with an uncertainty of 0.8 mas on polar motion and $0.2 \mathrm{~ms}$ on UT1, and predictions up to 1 year in the Weekly Bulletin A. The sub-Bureau also maintains a permanently revised EOP solution.

The Central Bureau produces and distributes four basic series of EOP. The first three, EOP(IERS) C 01, 02,03 , are intended for geophysical investigations at long, medium and short term. They give normal values at 0.05 year, five-day and one-day intervals starting respectively in 1846,1962 , and 1984 (UT1)/mid1992 (polar motion). The fourth one, C 04, is a continuous series at daily intervals since 1962; the series is slightly low pass filtered, so that it can be linearly interpolated rightaway. This solution is permanently updated. It is fit to all applications where an accurate model of the Earth orientation irregularities is needed. Monthly Bulletin B is an operational version of C 04 , based on the same data as Bulletin A.

\subsubsection{IERS Conventions}

The investigation of the variations of the Earth's rotation at all time scales is complicated by the mix of reference frame and modelling effects, and by the possibility of systematic errors, often at a level comparable with the amplitudes that are being investigated. A contribution of major importance in the unified analysis of independent observing programs is the formulation and the permanent improvement of a set of common models and standards to be used: reference systems, astronomical constants, models of local site displacements, etc, are treated in a homogeneous way by the IERS participants. They are published as the IERS Conventions, which serve as the conventional reference inside the IERS, but are also adopted by many users in space geodesy, geophysics and astronomy. The IERS Conventions follow in general the IAU and IUGG recommendations, except in the cases where the accuracy of observations makes it possible or necessary to adopt advanced modelling, as in the example of precession and nutation models. The analysis of VLBI and LLR observations led to the establishment of an empirical precessionnutation correction model to the IAU 1976 precession and the IAU 1980 Theory of Nutation, based on the Kinoshita-Souchay (1990) rigid Earth luni-solar and planetary nutations. This correction model is a part of the IERS Conventions (1996) that replace the IERS Standards (1992). 


\subsubsection{Atmospheric Angular Momentum}

The atmospheric parameters, which are related to Earth rotation, are archived, distributed to scientists, and made available to the users. In addition, the Sub-bureau intercompares the analysis data sets and assesses the skill of forecasts. Two intensive campaigns were held: SEARCH'92, during which atmospheric data sets were collected on a higher frequency basis than heretofore, in conjunction with the geodetic sets of Earth rotation parameters that were being collected at the same time, and a measurements campaign (CONT'94), held in January 1994. A major effort to analyse the state of the atmosphere in a consistent way was performed back to 1979 , with the eventual goal to produce historical data back to 1957 or 1958 . The reanalysis data are available by ftp.

\subsection{IERS PUBLICATIONS}

Weekly Bulletin A Earth rotation: Rapid Service, prediction.

Monthly Bulletin B Earth rotation, information on UTC time scale.

Annual Report Earth rotation, terrestrial and celestial frames of the IERS Reference System: combined solutions and analysis of individual results.

Special Bulletin C Announcement of the leap seconds in UTC.

Special Bulletin D Announcement of the value of DUT1 to be transmitted with time signals.

Technical Notes Reports and complementary information of relevance to the work of IERS on Earth orientation and the reference systems.

IERS Gazette Operational and technical information on IERS (electronic distribution only).

Bulletin A is prepared and distributed by the Sub-bureau for Rapid Service and Predictions. Other series are prepared and distributed by the Central Bureau. The mailing list includes about 800 institutes in 60 countries: space-geodesy and astronomical observatories, geodetic institutes, universities, time services.

\subsection{ELECTRONIC ACCESS TO IERS BUREAUX AND PUBLICATIONS}

\subsubsection{Central Bureau}

e-mail address: iers@obspm.fr

WWW: $\quad$ ftp://hpvlbi.obspm.fr/iers/ierscb.html

Earth rotation and celestial frame

Anonymous ftp: 145.238 .100 .7 (hpvlbi.obspm.fr)

$\begin{array}{ll} & \text { cd /iers/eop (resp. cd /iers/icrf) } \\ \text { WWW: } & \text { ftp://hpvlbi.obspm.fr/iers/ierscb.html }\end{array}$

Terrestrial frame

e-mail address: itrf@ign.fr

Anonymous ftp: 192.33 .147 .230 (schubert.ign.fr)

WWW: $\quad$ http://schubert.ign.fr/CIAG/index.CIAG.html

2.5.2. IERS Conventions (1996), IERS Technical Note No 21

Anonymous ftp: 192.5.41.22 (maia.usno.navy.mil)

cd conventions

2.5.3. Sub-Bureau for Rapid Service and Predictions

e-mail address: ser7@maia.usno.navy.mil

Anonymous ftp: 192.5 .41 .22 (maia.usno.navy.mil)

WWW: http://maia.usno.navy.mil

2.5.4. Sub-Bureau for Atmospheric Angular Momentum

e-mail address: salstein@aer.com

Anonymous ftp: climon.wwb.noaa.gov

WWW: $\quad$ http://www.aer.com

\section{WORKING GROUP ON EARTH ROTATION IN HIPPARCOS REFERENCE FRAME}

The collection of optical observations of time/latitude from selected observatories continued successfully; the data obtained with 46 instruments at 29 observatories, covering the interval 1899.7-1992.0, have 
been gathered so far. The observed values (re-calculated into a unique system of astronomical standards and corrected for plate tectonic motions, certain instrumental constants, deformations of the apparent almucantar, short-periodic zonal tide variations in UT and ocean tide-loading variations of the local vertical) were used to estimate polar motion, UT1 and celestial pole offsets at 5-day intervals plus several hundred auxiliary parameters. 11 different solutions were made, with the number of participating instruments and observations increasing from 7 to 46 and from 739754 to 4019218 , respectively (Vondrák, Ron \& Cepek 1993, Vondrák \& Ron 1994, Vondrák, Ron \& Čepek 1994, Vondrák, Ron, Pešek \& Cepek 1995 , Vondrák \& Ron 1996). To be able to solve such large system of normal equations $(\sim 30$ thousand parameters) with sparse matrix, a special procedure based on modified Cholesky decomposition was designed (Cepek 1994).

Thanks to the newly established cooperation with the Hipparcos Science Team on the link of Hipparcos catalog to extragalactic reference frame (Vondrák 1996), the WG obtained relevant parts of preliminary Hipparcos catalogs $\mathrm{H} 30, \mathrm{H} 37$ and $\mathrm{H} 37 \mathrm{C}$, and used them in the last six solutions. The one referred to $\mathrm{H37C}$ was used, together with VLBI solution of Earth orientation parameters, to determine four of the six parameters defining the tie between optical and radio reference frame. They were submitted to the Hipparcos Science Team to be combined with other determinations. The common feature of our last solution is the accuracy of polar motion slowly improving from 0.03 " around 1900 to 0.01 " in 19801985 and dropping to 0.02 " around 1990. A very similar pattern is seen in celestial pole offsets, but their standard errors display a significant semi-annual variation, due to correlations between the two components. UT1 determination is characterized by standard errors of $0.0020 \mathrm{~s}$ in 1956 , slowly decreasing to a minimum of $0.0008 \mathrm{~s}$ in 1983 and, after 1988, rapidly increasing to 0.0015 in 1991.

\section{REPORTS FROM THE INDIVIDUAL OBSERVATORIES AND INSTITUTIONS}

\subsection{U. S. NAVAL OBSERVATORY, WASHINGTON D.C., USA}

\subsubsection{Distribution of Earth Orientation Information}

The U. S. Naval Observatory (USNO), as a partner in the National Earth Orientation Service (NEOS) with the National Oceanic and Atmospheric Administration (NOAA), continued to function as the Subbureau for Rapid Service and Prediction of the IERS. Weekly publication of IERS Bulletin A was sent to approximately 300 users by mail and 200 users by e-mail. NEOS Annual Reports for 1993, 1994 and 1995 were distributed to users. The NEOS Earth orientation bulletin board now has 65 regular users. Earth orientation data information was also made available by anonymous ftp and the NEOS home page.

Weekly analysis of Earth orientation data derived from GPS orbits was continued by Luzum \& McCarthy. The information is provided to the IGS for distribution to its users. A software, combining different GPS Earth orientation series, provides a single GPS combination series in order to account for changes in the system and improvements in the data. A special prediction file to be used by all participating in the IGS is produced routinely. The polar motion data from the Institute of Applied Astronomy (St. Petersburg) was analyzed and is now used routinely in the weekly rapid service.

\subsubsection{Very Long Baseline Interferometry}

The NEOS VLBI network created from the merger of the NOAA IRIS and the USNO NAVNET networks continued routine operation. In the three-year reporting period a total of 175 NEOS experiments of 24hour duration have been fully processed creating a data base of 164,281 delay observations. The processing turn-around times for these experiments averaged less than seven days after acquisition of data. The accuracy appears to be $\pm 0.3 \mathrm{mas}$ in $x, \pm 0.3 \mathrm{mas}$ in $y$, and $\pm 0.015 \mathrm{~ms}$ in UT1-UTC. A proposal for the NEOS consortium of USNO, NASA and NOAA to act as the coordinating center for VLBI in the International Earth Rotation Service (IERS) was accepted.

Current NEOS operations consist of one weekly 24-hour duration NEOS-A observing session per week for Earth orientation. The operational NEOS-A network currently includes the VLBI stations at Gilmore Creek, Alaska, Kokee Park, Hawaii, Wettzell, Germany, Fortaleza, Brazil, and Green Bank, West Virginia. The USNO also has responsibility for the NEOS UT1 Intensive Series. These short duration single baseline observations are being continued using the same scheduling and observing procedures as before to ensure continuity with previous results. Current intensive operations involve 16 observations conducted on the Green Bank 20-meter to Wettzell baseline. These observations are conducted at the same sidereal time each day, except during the NEOS 24-hour sessions. Roughly every 3 months the 
sidereal time of the intensives is shifted to keep their solar time centered around 18:00 UTC. There were 1174 intensives observed during the reporting period, using a total of 18,188 observations with a postfit wrms residual scatter of \pm 16 picoseconds for the delay, and \pm 117 femtoseconds/seconds for the delay rate. UT1 formal errors for these sessions ranged between \pm 0.010 and $\pm 0.020 \mathrm{~ms}$.

Monthly NEOS-B sessions sponsored by NOAA were performed during much of 1994 to improve the terrestrial reference frame. These sessions were terminated at the end of 1994 due to reductions in the NOAA VLBI budget, with the last NEOS-B session being on October 6 and 7, 1994.

\subsubsection{Prediction of Earth Orientation}

The prediction of the corrections to the IAU nutation theory was modified, taking into account the most recent observations. Predictions of polar motion, UT1-UTC, nutation and UT were provided to the IERS. The polar motion algorithm was also modified to include a semiannual term which improved the prediction accuracy by approximately $20 \%$.

\subsubsection{Reference Systems}

Fey \& Eubanks participated in an IAU working group on reference frames sub-group established for the purpose of establishing a radio reference frame. Work was begun on various ways to make source structure information obtained from the VLBA images available to the CALC/SOLVE software. This includes adapting source code provided by the Jet Propulsion Laboratory.

Both the S- and the X-band images of radio reference frame sources are now available. A Radio Reference Frame Image Database (RRFID) WWW page was created to allow easier access to the VLBI radio images of reference frame sources.

An agreement with NASA was reached to schedule eight Celestial Reference Frame (CRF) experiments in 1995. Two were dedicated to the northern hemisphere, four to the 'Mid-South' including the St. Croix and Mauna Kea VLBA stations, and two were dedicated to the 'Deep South', including DSS45 in Australia. In the southern hemisphere observations were continued at the same pace in 1996.

The Navy 1996-4 reference frame is the frame used in the operational USNO Earth orientation solutions. It was prepared using 1460363 delay and delay rate observation pairs from 1949 observing sessions (complete through January 2,1996 ), with a weighted rms residual scatter of \pm 31.93 picoseconds for the delay observations and \pm 94.07 femtoseconds per second for the delay rate data.

\subsubsection{Analyses of Earth Orientation Observations}

Luzum \& McCarthy continued the study of the application of atmospheric angular momentum data to the estimation of Earth orientation. Corrections to the main zonal tide components in the variation of the length of day were derived in the process and used to derive corrections to the zonal tidal coefficients for UT1-UTC. Observations of nutation were investigated by McCarthy and Luzum. Improved model coefficients were determined. Luzum also completed work on extending the NEOS combination Earth orientation time series back to 1976, as an a priori standard for VLBI analysts. McCarthy \& Luzum investigated various ocean tide models by comparing the predicted variations in length of day with observations. Archinal continued his investigations on the use of the GEODYN/SOLVE software, with which it could be possible to do direct combination of SLR/VLBI/GPS solutions.

\subsection{JET PROPULSION LABORATORY, PASADENA, USA}

Accuracies of \pm 0.3 mas for polar motion and $\pm 0.03 \mathrm{~ms}$ for UT1 are now being achieved by the modern space geodetic techniques. Annual combinations of independent Earth orientation measurements have continued to be generated. Estimates of the atmospheric excitation of Earth orientation, provided by the routine analyses of global weather data, together with the modern Earth orientation measurements have allowed new insight into atmospheric and non-atmospheric excitation of Earth orientation variations. It was shown that changes in the length of day (LOD) at seasonal and higher frequencies are dominated by the exchange of angular momentum between the atmosphere and the solid Earth except at tidal periods and are coherent down to 8 days, with lack of coherence at shorter periods caused by the declining signalto-measurement noise ratio in both the AAM and LOD data types. The correlation between atmospheric angular momentum (AAM) and LOD suggests that AAM analysis and forecast fields may improve nearreal time estimates and prediction of Earth rotation changes. Using GPS measurements along with the non-axial components of the AAM $\chi$-functions computed from general circulation models of the 
atmosphere, it has been shown that atmospheric wind and pressure fluctuations were largely responsible for exciting the observed rapid polar motions.

Subdaily Earth orientation changes have been detected by both the GPS and VLBI observing programs at JPL and have been interpreted in terms of ocean tidal effects on the Earth's rotation. The angular momentum carried by the ocean tidal current and sea level height variations can be exchanged with the angular momentum of the solid Earth. Formulae to compute changes in the Earth's orientation from ocean tidal angular momenta have been summarized and applied to results of the Brosche tide model. Discrepancies between observed Earth orientation changes in the long period tidal band, particularly at the fortnightly tidal frequencies, and those predicted by ocean tide models suggest the need for improved long period ocean tide models.

The source of the intraseasonal "0-50 day" oscillation has been explored by analyzing LOD data in concert with AAM results. Findings indicate that two intraseasonal oscillations exist in the Earth-atmosphere system: a tropical 50-day oscillation associated with the convectively driven Madden-Julian wave, and a mid-latitude 40-day oscillation associated with the interaction of non-zonal flow with topography.

At the longer period interannual time frame, the El Niño/Southern Oscillation (ENSO) phenomenon has been linked to LOD variations and the bimodality of both LOD and Southern Oscillation Index (SOI) fluctuations has been established. Significant variability is centered at $\sim 4.2$ years and is associated with the 'traditional' ENSO cycle; in addition, a distinct quasi-biennial component (at $\sim 2.3$ years) is observed. A case study of the 1982-83 ENSO event indicates that the stratosphere is an important contributor on these time scales and that $90 \%$ of the LOD variance can be explained by the atmosphere. Examining the latitudinal redistribution of angular momentum within the atmosphere, we discovered a slow, global-scale poleward AAM propagation that originates in equatorial regions, where westerly anomalies lead the main ENSO anomalies by nearly two years. Coherent signals penetrate to latitudes higher than $60^{\circ}$ in both hemispheres, where they lag behind the ENSO cycle by fours years. This propagating V-like structure is evident in atmospheric general circulation models (GCMs) results that are driven by realistic sea surface temperatures (SST); however, it is absent from GCM runs made with climatological SST's. Examining LOD series back to 1880 , high correlation between LOD and SOI is obtained from about 1930 to the present, indicating that LOD can be used as a proxy-index for interannual variations in global wind systems after 1930 .

Decadal fluctuations in the Earth's rotation are also being studied. The magnitude of the topographic torque on the Earth's mantle due to just the spherical harmonic degree 2 component of the core-mantle boundary topography has been investigated and found to contribute about $20 \%$ to the observed decadal polar motion signal. An on-going study of the effect of earthquakes on global geodynamic properties of the Earth has resulted in predictions of the effects of earthquakes on the Earth's rotational energy (Chao and Gross 1995), gravitational energy (Chao et al. 1995), and polar motion (Chao et al. 1996).

The analysis of lunar laser ranging (LLR) data influences a broad spectrum of studies in astronomy and related sciences. The LLR-determined value of the principal term in the Earth's gravity field is $G M_{E a r t h}=398600.443 \pm 0.004 \mathrm{~km}^{3} / \mathrm{sec}^{2}$, the value of the tidally driven secular acceleration of the Moon is $-25.88 \pm 0.5 \mathrm{arcsec} / \mathrm{cy}^{2}$ corresponding to an increase of $3.80 \pm 0.07 \mathrm{~cm} / \mathrm{yr}$ in the mean distance of the Moon. The correction to the IAU-adopted precession constant is $-3.2 \pm 0.3 \mathrm{mas} / \mathrm{yr}$, giving the lunisolar precession constant as $50.3846 \mathrm{arcsec} / \mathrm{yr}$ at $\mathrm{J} 2000$. The amplitude of the 18.6 -year motion of the Earth's pole in space needs to be increased by nearly 3 mas. A joint analysis of LLR and VLBI data gives precession a correction of $-3.00 \pm 0.20 \mathrm{mas} / \mathrm{yr}$ and an obliquity rate correction of $-0.20 \pm 0.08$ mas $/ \mathrm{yr}$.

The JPL Celestial Reference Frame is based on VLBI observations of extragalactic radio sources. As a participant in the IAU working group on reference frames, JPL contributes to the adoption of the extragalactic radio frame as the IAU's fundamental celestial frame. It also works closely to tie the radio frame with the Hipparcos catalog. VLBI data collection was upgraded to the MkIIIa recording system. VLBI observable modeling was improved in several areas, namely in tidal effects, troposphere and source structure. The troposphere model was improved with upgrades to Lanyi's mapping function as well as the inclusion of the alternative models of Herring and Niell. The benefits of WVR have been demonstrated using troposphere calibration techniques for VLBI. We now include thermal expansion of the antenna structure. A collaborative effort with our French colleague has begun to yield results in the modeling of source structure. This collaboration also produced new estimates of precession and nutation. Intercomparisons of the JPL and GSFC VLBI software were made to verify their accuracy. Based on the delays and rates from two observing sessions, our modeling was reconciled at the $1 \mathrm{psec}$ and $1 \mathrm{fsec} / \mathrm{sec}$ 
levels respectively. An end-to-end test of modeling and parameter estimation was made by comparing celestial frames produced from a common set of data. These frames agreed with a wrms scatter of $\leq 0.21$ mas, relative rotations of $\leq 0.14$ mas and biases in RA and declination of $\leq 0.02$ mas. Comparisons using independent data sets produced agreement at the 0.3 mas level.

\subsection{ACTIVITIES OF RESEARCH WORK RELATED TO EARTH ROTATION IN CHINA}

\subsubsection{Instrumentation and Observations}

The Urumqi VLBI station was operated regularly since November 1993. This station is equipped with a 25m antenna and a MKIIIA terminal (Ye \& Qian 1994). From 1994 the Beijing SLR station began to operate routinely (Wang T.Q. 1994). Now the Chinese SLR network consists of Shanghai, Wuhan, Changchun and Beijing stations (Xia \& Xia 1993). In November 1994 the West Pacific Laser Tracking Network (WPLTN), which contains SLR stations in Japan, Australia, China and Russia, was set up. A new SFUR laser with pulse width of $100 \mathrm{ps}$ was equipped at Shanghai station in October 1995. 13 satellites are routinely observed with a ranging precision of $3-5 \mathrm{~cm}$ by the Chinese Network. A Chinese transportable Laser Ranging System is assembled and tested (Xia et al. 1994).

The data of the IGS'92 campaign in China have been processed. The accuracy of the global baselines is $5 \mathrm{~mm} \pm 8.35 \mathrm{ppb}$, the accuracy of orbit determination is better than $2 \mathrm{~m}$, the accuracy of polar motion is about 1 mas (Wang J.X. et al. 1994). The second and third GPS campaigns were held in AugustSeptember 1994 and June 1996 respectively. In January 1995 Shanghai GPS station installed a Rogue SNR 8100 receiver. Now there are 22 GPS stations in the Chinese network.

At the end of 1994 a $1.2 \mathrm{~m}$ telescope with equipment of adaptive optics was completed at Yunnan Observatory. The pointing precision is about 1 arcsecond. The laser system is under construction for the work of SLR and LLR.

Optical astrometrical observations are still conducted at many stations in the world for determining the positions of stars or detecting the variations of plumb line due to local geophysical causes; there are about 35 instruments continuing the observation. Shanghai Observatory was assigned by Commission 19 at the 20th General Assembly of IAU as a center to collect and analyze optical observations.

\subsubsection{Determination of Earth Orientation Parameters}

As one of the analysis centers of the global VLBI, SLR and LLR data, Shanghai Observatory continues to present the analysis results to the IERS. Chinese Joint Universal Time and polar coordinates related to mean origin of epoch 1968.0 were used by Chinese geodesists, geoplyysicists, astronomers etc.. A homogeneous series of Earth orientation parameters referred to the FK5, IAU 1980 nutation series and IAU 1976 astronomical constants was worked out. The comparison between this series and ERP(BIH) 87 C02 was made (Liao et al. 1993; Lu et al. 1995; Jin et al. 1995a). The corrections to the Standard Time published by Institute of Metrology for Time and Space, Russia during 1953-1964 were calculated (Liao et al. 1994), and the values of Chinese longitude origin with respect to different polar origins (such as CIO, mean pole of epoch and mean pole of epoch 1968.0) were recalculated taking into consideration the Earth solid and ocean tides (Jin et al. 1995b). The Earth orientation parameters were determined by using the data of IRIS-A network based on different relativistic models. Comparisons with other series were made; it is shown that the differences are significant (Yang et al. 1994). The direct effect of mantle inelasticity on the effective Love number $k$ and the indirect perturbation due to the response of the inelastic Earth to the equilibrium ocean tide are discussed by using the theoretical period of the Chandler wobble as the constraint to estimate the parameter of the absorption band model. Their effects on the scaling factor $k / C$ of the zonal tides are also analysed. The series of the tidal variation in universal time UT1 is calculated for an Earth with dynamical ocean, inelastic mantle and fluid core (Zhu 1994).

\subsubsection{Studies of Polar Motion}

During IGS' 92 campaign, seven Polar Motion series provided by GPS Processing Centers were analysed using spectral analysis, least squares adjustment and $F$-test (Huang et al. 1995). The spectrum of EOP (SHA) series has been analysed. Some main periodical terms of both polar motion and Earth rotation were found from this series, which shows that the series can be reliably applied to astronomic and geophysical researches. The changes in high frequencies are also shown (Zhu \& Zhu 1993). A positive correlation between the velocity and the amplitude of polar motion, and a negative correlation between the velocity and the period of polar motion is found; El Niño always happened in the year when the period of polar 
motion was short (Qian 1995). The geodetic excitation derived from new highly accurate polar motion data (Space 93 series) and the atmospheric excitation function supplied by Japan Meteorological Agency are used to investigate the nonseasonal oscillation. A relatively strong $40-60$ days oscillation is revealed from these data series. Statistic analyses show that the geodetic excitation is related to the atmospheric pressure change on nonseasonal time scale and it is driven by surface air pressure change, playing a role at periods less than 140 days (Qian \& Gu 1994; Gu 1995b).

\subsubsection{Short Period Fluctuations of Earth Rotation, Atmosphere, Ocean and Solar Activities}

A positive correlation between LOD in the interannual time scale and Sea Level Change (SLC) in the equatorial area of the eastern Pacific, and a negative correlation between LOD and SLC in the area of the western Pacific is found (the mean sea level in the Pacific has been rising with the rate of $1.8 \mathrm{~mm} /$ year for the recent 30 years). A new model for the interaction between equatorial ocean and Earth rotation is developed (Zheng \& Chen 1993, 1994, Zheng et al. 1995, 1996, Yu \& Zheng 1996). The torque produced by the solar wind on the Earth might be considered as one of the most possible contributions to annual fluctuation in $\operatorname{LOD}$ (Gu 1993, 1994, 1995a, 1996, Gu et al. 1995, Yang et al. 1994). The latitude residuals at Washington have a significant correlation, in interannual time scale, with the Southern Oscillation Index. It might be considered as an evidence that the plumb line variations are related to the large scale movement of the Earth atmosphere-oceanic system ( $\mathrm{Li} \mathrm{1995).} \mathrm{It} \mathrm{is} \mathrm{shown} \mathrm{that} \mathrm{the} \mathrm{atmosphere} \mathrm{may}$ explain a part of non-tidal high frequency variation in LOD up to 30 days and that the high frequency variation of polar motion up to 30 days are mainly excited by the atmosphere (Xie \& Zheng 1996).

\subsubsection{Secular Variations in the Rate of Earth Rotation}

732 Chinese lunar records are used to study the secular variation of the Earth rotation. They fill in the gap of data from the $3 \mathrm{rd}$ to the 8 th century and prove an apparent change of the nontidal term about 5 th century (Liu 1994). A Japanese ancient record of the annular solar eclipse on November 17, A.D. 1183 was studied. The value of $\Delta T$ found implies an increase of LOD of about $1.6 \mathrm{~ms} / \mathrm{cy}$ (Han 1994). 34 timing solar eclipse observations recorded in the Twenty-Four Histories during the period from $\mathrm{BC}$ 2nd to AD 8th century were analyzed; the tendency of the secular change in the rotation of the Earth was probed (Zhang \& Han 1995). The non-tidal effect in the secular variation of the angular velocity of the Earth rotation on the observed average values was estimated, and the secular change of the Earth's principal moment of inertia $C$ found; the result is in contradiction to the expanding Earth hypothesis (Yang \& Groten 1995). The best historical material comes from two periods: 7 th century $\mathrm{BC}$ to 2 nd century $\mathrm{AD}$ and 9 th to 12 th century $\mathrm{AD}$. Since the results from the two parts are apparently different, the weak link of ancient records from 3rd to 8 th century is very noticeable. 732 records of lunar occultations and approaches before $960 \mathrm{AD}$ were chosen to study the Earth's rotation by a new method called "Time Window (Really Visible Time)". The mean acceleration obtained is quite consistent with other sources. The discovery needs further investigation (Wu \& Liu 1993). The ice sheet mass changes in Antarctica and Greenland (that correspond to about $41 \%$ of mass changes of all ice sheets on Earth) are insufficient to explain the non-tidal component of Earth rotation. The global sea level change would be more believable; postglacial rebound would be even more possible (Yang 1996).

\subsubsection{Relationship Between Earth Rotation and Earthquakes}

The relation of the decadal, interannual and seasonal changes in the Earth rotation with the seismic activity in China was statistically analysed. It is concluded that the stress caused by the variations of Earth rotation might trigger the seismic activity, and that the data on Earth rotation might provide a basis for the prediction of seismic activities (Zhou \& Zheng 1994). Interrelation between major earthquakes that occurred in southwest China during the last three decades and the variation of tidal force by the Moon and the Sun was studied. The results show that the dates of the earthquakes are related to the variation of horizontal east-west component of the force (Han \& Tian 1993; Han et al. 1994).

\subsubsection{Methods for VLBI, LLR and SLR Data Reduction}

The VLBI relativistic time delay and delay rate models with an accuracy of $1 \mathrm{ps}$ and $10^{-3} \mathrm{ps} / \mathrm{s}$ were discussed (Zheng et al. 1993; Yang 1993c). For computing gravitational effects in processing VLBI data, the principal difference between the deflection method and differential "light-time" method is caused only by the variation of the velocity of radio signal passing through the solar gravitational field (Ping 1993). The structure of SOLVK software for VLBI is analysed and three modifications have been performed 
(Wang G.L. et al. 1994). The values of atmospheric delay for different mapping functions were calculated by using the atmospheric parameters at Shanghai, Kunming and Urumqi. It was shown that the deviation of "Marini" mapping function from the "Chao" and the "CfA-2.2" mapping function respectively was relatively large. It is possible that the inconsistency between these models mainly come from the "Chao" model. Therefore, it is necessary to make a better revision to the model of atmospheric delay (Yang 1993a; 1993b). The new mapping function forms of the tropospheric delay have been derived for the standard atmospheric model and Hopfield atmospheric profile, respectively. The accuracies of these new models are better than $1 \mathrm{~cm}$ for an elevation angle lower than $2.5^{\circ}$ over a wide range of meteorological conditions (Yan \& Ping 1995).

The observations at all LLR stations in the world are used to find the corrections to the solar and lunar orbital elements (Bai et al. 1994). Using laser ranging observations of the LAGEOS satellite in 1985-1990, the rate of change of the baseline vector between QUINCY and MNPEAK stations was estimated using a semi-dynamic and semi-geometric method. The standard error of the baseline length obtained from one day observation is $3.0 \mathrm{~mm}$ or $3.4 \times 10^{-9}$ (Zhu et al. 1994). A new robust estimate method in SLR data preprocessing is presented. The robust estimator has $50 \%$ breakdown point and is free from the leverage points. The new method has good performance for rejecting outliers in SLR data and it makes the preprocessing automatic (Jia et al. 1994). Analyses show that the SLR observations belong to $p$-norm distribution with inconstant $p$ value; adaptive $L p$ estimation gives more robust solution than least-squares estimation (Wu et al. 1996). A method to preprocess the SLR data through computer screen is presented. The method, function, and a practical application of the software were described (Zhang 1993).

\subsubsection{Miscellaneous}

All optical time/latitude observations in China, referred to the BIH system, have residuals with a similar annual term. These annual variations can be partly explained by anomalous refraction (Gao 1994). The relativistic effects in precession/nutation were discussed, the geodesic precession and nutation being the largest ones (Li \& Huang 1993). The contribution of the redistribution of the density in the Earth's interior (especially in the core) to the change of the flattening at the core mantle boundary (CMB) is marginal, and the calculated value of the flattening at the CMB can be in good agreement with the VLBI observed value if the fact that the angular velocity of the Earth rotation has undergone tidal evolution is taken into account. A set of recommended values of the dynamical parameters of the Earth for computing Earth's forced nutation series are also presented (Zhang \& Xia 1994). From the first two years of TOPEX satellite altimetry the rate of rise in global mean sea level and in the regional mean sea level near the Shanghai coast were estimated as $2.9 \mathrm{~mm} / \mathrm{yr}$ and $3.9 \mathrm{~mm} / \mathrm{yr}$ respectively. These results are consistent with the NASA's ones and in reasonable agreement with the results from almost 30 years of tide gauge data of the East China Sea (Huang \& Dong 1996). An instantaneous plate motion model (SPMM1), consisting of 10 Euler vectors between 5 plates, was derived. Comparison with the models RM2 and NUVEL-1 indicates that SPMM1 is roughly consistent with the geological models. (Sun \& Zhao 1994). A 1-2 cm eastward movement of Shanghai was detected from VLBI experiments (Ye et al. 1994), the same movement was detected from SLR observations, but the direction of movement is about 30 degree southeast.

\subsection{NATIONAL ASTRONOMICAL OBSERVATORY, JAPAN}

The VLBI antenna at Mizusawa Astrogeodynamics Observatory became operational in 1993 and participated in the IRIS-P until the end of 1994 . In 1995 a project to deploy K4 VLBI recorders to existing radio observatories and set up a new VLBI network started. One recorder was placed at the Urumqi Astrophysical Observatory in China and test observations between Mizusawa and Urumqi were performed in the same year. Another K4 was placed at Simeiz station of the Crimean Astrophysical Observatory in Ukraine in 1996 and the observation between Mizusawa and Simeiz was also performed. All the data were correlated at Mizusawa with a NAOCO which is based on an architecture different from both Mark III and K3. Domestic geodetic observations among Mizusawa, Kashima, Kumamoto and Kagoshima were also processed with this correlator. Experimental VLBI observations in the K-band were performed between Mizusawa and Kashima and between Mizusawa and Kagoshima. The experiments in the K-band and in S/X-bands in adjacent days showed good repeatability, namely, $1 \mathrm{~cm}$ in the horizontal components. Search for radio sources appropriate for geodetic observations in the K-band is under way.

Software for the correlation with the NAOCO and the bandwidth synthesis was completed and has been used for both geodetic and astrophysical observations. It has become possible to export results of 
K-4 based observations in the Mark III database format.

Global analysis of VLBI data was done and reported to IERS. Abe et al.(1994,1996) and Ooe et al.(1995) discussed tidal evolution of Earth rotation and the Earth-Moon system quantitatively, based on various models of ocean-continent configuration and interpreted paleontological rotation up to $600 \mathrm{Ma}$ b.p. Sato et al.(1994) analysed gravimetric data obtained with three superconducting gravimeters in Japan and strongly suggested that low $Q$-values of the free core nutation obtained in preceeding studies were due to the unconserved mass in the ocean tidal models. The modification of the ocean model to conserve the mass resulted in a good agreement with the $Q$-value obtained from VLBI. Gravimetric observations with superconducting gravimeters have continuously been carried out at Esashi near Mizusawa and Syowa station in Antarctica for observations of motions of the liquid core and the inner core. Another one is going to be placed in Australia for the same purpose in 1996.

\subsection{ROYAL OBSERVATORY OF BELGIUM, BRUSSELS}

\subsubsection{The Earth's Rotation}

The stability in frequency and amplitude of the Madden-Julian oscillation confirms the presence of several spectral concentrations between 40 and 60 days. Correlations in phase and amplitude between the 50-day oscillation of the Earth rotation, the Effective Atmospheric Angular Momentum (EAAM), Wolf numbers and solar corona indices were investigated (Djurović, Pâquet and Billiau 1994; Djurović and Pâquet 1994; Zhen-Nian, Djurović and Pâquet 1995). The existence of the 4-month oscillation has been confirmed both in geophysical and solar data series; in the EAAM and Earth rotation, the amplitudes are slightly above those observed in the Madden-Julian oscillation (Djurović and Pâquet 1994, 1995). At longer periods, same correlations between solar activity and Earth rotation are observed:

- the quasi-biennial oscillation has been found in the green corona index, the Wolf number, the geomagnetic field, the EAAM and universal time (Djurović and Pâquet 1993),

- the Earth rotation exhibits an oscillation close to 5.5 years; it has been shown that its origin could also be in solar activity variations but the interface is not the atmospheric angular momentum but rather the Earth magnetic field (Djurović and Pâquet 1996).

\subsubsection{Precession/Nutation}

The precession, obliquity rate and nutations for a rigid Earth have been computed analytically; the gravitational torque by the Moon, the Sun, and the planets on the Earth have been considered. The computations include both direct and indirect effects. The $J_{2}$-tilt effects are also considered; they are due to the fact that the Earth's equatorial bulge induces additional perturbations on the Moon which in turn induces variations in the lunar attraction. The rigid Earth nutations are then multiplied by a non-rigid Earth transfer function; the comparison with observations shows that the geophysical model still needs to be improved.

The Free Core Nutation (FCN) as computed theoretically from the PREM rheological properties and the hydrostatic equilibrium is about 458 sidereal days in inertial frame; on the other hand, the tidal and nutation observations have been used together (Defraigne et al. 1994, 1995) to find the FCN period of about 433 sidereal days. It has been suggested in 1986 by Herring et al. that an increase of the equatorial radius of the core of only about 500 metres would induce such a decrease of the FCN period. This change of the core radius can be explained by considering violation of the hydrostatic equilibrium; Defraigne et al. (1996) have shown that the mantle-flow models favour an increase of the equatorial radius of the core in agreement with the excess CMB ellipticity deduced from observations of the FCN period.

The dynamical flattening $H=(C-A) / C$ associated with the hydrostatic equilibrium case applied on model PREM does not match the value derived from the observed precession rate (about $1 \%$ difference, as shown in Dehant \& Capitaine 1996). However, the variations of the dynamical flattening as computed from current mantle tomography density heterogeneities (Dehant \& Capitaine 1996) do not change the PREM dynamical flattening in the right direction; Dehant \& Capitaine also tried to use the mass anomalies given by the Ricard et al. (1993) model, which does not solve the difference either. It is possible to reconcile the difference by considering, in addition to the observed lateral heterogeneities, the mass anomalies associated with the boundary topography (displacements induced by the present mantle circulation). Due to isostasy at all the chemical boundaries and to partial compensation at the $670 \mathrm{~km}$ - depth boundary phase transition (Defraigne \& Dehant 1996), the effect of hydrostatic equilibrium violation on 
$H$ is only related to masses located either far away from the mentioned boundaries, or on the exothermic phase transition boundaries. Defraigne (1996) has found a model which agrees with the CMB flattening deduced from the VLBI observations, and with the observed $J_{2}$ and $H$ values, starting from Su et al. (1994)'s tomography model readjusting the lower mantle density anomalies. It includes an exothermic phase transition at $400 \mathrm{~km}$ depth and an endothermic phase transition at $670 \mathrm{~km}$. Dehant (1996) is now working on incorporating this model in the non-rigid Earth nutation computation. The atmospheric pressure torque acting on the Earth has been computed (Dehant et al. 1996), showing the necessity to account for the gravitational torque of the atmosphere on the Earth.

\subsubsection{IERS Standards}

Dehant \& Mathews have recomputed the tidal displacements (radial and transverse) of a station and the tidal contributions to the geopotential coefficients. This work is included in the publication of the IERS standards (IERS Conventions 1996).

\subsection{INSTITUTE OF ASTRONOMY \& STERNBERG ASTRONOMICAL INSTITUTE, MOSCOW, RUSSIA}

During the last few years Avsuk, Rykhlova, Gerasimov \& Nesterov carried out an investigation of the rotation of non-uniform Earth with a fluid center. Theory developed so far is based on three fundamental tenets:

- two reference frames are introduced: the stationary one, tied to the mean ecliptic, and the moving one, attached to the body being studied,

- the Earth-Moon barycenter moves around the Sun along the instantaneous ecliptic with a oneyear period. At the same time the Earth revolves around the barycenter along an elliptical orbit inclined by $5^{\circ}$ to the ecliptic, with a one-month period. As a consequence, the Earth's center of mass is perturbed, the amplitude of perturbation depending on the difference of the Earth's and Sun's ecliptic longitudes. The amplitude changes with a period of $412-437$ days, close to the observed value for the basic period of polar motion,

- existence of the inner Earth core, suspended in a liquid outer core, facilitated development of refined Earth structure models. Displacement of the inner core in response to perturbations may reach a few hundred meters. Such an effect will certainly lead to displacement of the origin of the reference frame attached to the Earth's center of mass. Observed components of the displacement $x, y$ show congruity with the values computed from components of the tidal force.

Based on the nonhydrostatic model of the Earth computed by Chujkova, Zharov calculated the nutation amplitudes using the modified Sasao-Okubo-Saito theory. After correcting this theory and taking into account the values of the second order he obtained the FCN frequency equal to -1.002334 cpsd (very close to the observed value). The dynamical ellipticity of the outer core is equal to 0.002686 . After taking into account the effect of the atmospheric tides the ellipticity is increased and becomes 0.002695 . The atmospheric tides together with oceanic tides and the effects of anelasticity in the mantle can explain the observed out-of-phase corrections to the nutation amplitudes.

The laser gyroscope has been tested. The first results were obtained, but the accuracy of measurements was still low due to the low power of the laser and bad quality of the resonator's mirrors. After a change of the laser and mirrors high quality data are expected.

\subsection{MAIN ASTRONOMICAL OBSERVATORY, KIEV, UKRAINE}

Traditionally, the Main Astronomical Observatory takes part in IERS as an analysis center of SLR and VLBI. Kudlay has considered some problems of the non-linear theory of the Earth rotation. It was shown that non-linear terms can significantly peturb wobble parameters (Chandler mode frequency shift and variability etc.) and even to cause core's irregular motion. Bolotin in cooperation with French colleagues have proposed new approach for estimating diurnal variation of the Earth's angular velocity vector. Kuryanova \& Yatskiv have proposed a new method for deriving compilation catalogues of extragalactic radio sources. Rudenko \& Capitaine estimated pole coordinates and universal time at submas accuracy from satellite laser ranging data, using the non-rotating origin. Rudenko obtained some of the analysis of the geosynchronous satellites observations. Korsuń, Kurbasova \& Vondrák compared the new global solution of Earth orientation parameters by optical astrometry with other determinations in 1900-1990. 
Mironov, Shvalagin \& Kablak considered the validation of the IERS standard troposheric model as SLR instrumental precision has been improved to the the centimeter and even subcentimeter levels.

\subsection{PARIS OBSERVATORY, FRANCE}

Two Journées Systèmes de Référence spatio-temporels have been organized in Paris (June 1994) and in Warsaw (September 1995) by the Département d'Astronomie Fondamentale of the Paris Observatory, the latter in cooperation with the Space Research Center of Warsaw (N. Capitaine, ed. 1994, N. Capitaine, B. Kołaczek \& S. Débarbat, eds. 1996).

The theoretical aspects of nutation and reference frames have been studied (Capitaine 1995) as well as the questions of astronomical units and constants in a relativistic framework (Capitaine \& Guinot 1995).

Estimation of precession and nutation corrections have been derived from 1980-1993 GSFC VLBI data, taking into account correlations between the long periodic and linear terms through theoretical constraints (Souchay, Feissel, Bizouard, Capitaine \& Bougeard 1995). This provides estimates of corrections due to the imperfections in the modeling for the largest components of nutation and for the linear trends corresponding to a precession in longitude $(-3.31 \mathrm{mas} / \mathrm{yr})$ and a rate in obliquity $(-0.26 \mathrm{mas} / \mathrm{y})$ relative to the IERS Celestial Reference System. Celestial pole offsets in preliminary Hipparcos reference frame as determined by optical astrometry (1899.7-1992.0) have been combined with VLBI results and the corrections to long-periodic nutation terms found (Bizouard, Capitaine, Ron \& Vondrák 1996).

The high frequency variations of the instantaneous angular velocity vector have been derived from a 15-day period of VLBI observations (from NASA-R\&D + NEOS-A) with very short temporal resolution (Bolotin, Bizouard, Loyer \& Capitaine 1996).

\subsection{ASTRONOMICAL OBSERVATORY BUCHAREST, RUMANIA}

The observations made with the Bucharest visual transit instrument in the period 1957-1990 were analyzed; long period terms of $27.8,18.6,13.9,12.5,11.1,9.3$ and 6.4 years were detected in UT1. The influence of the terrestrial rotation on the motion of artificial satellites was investigated in the case of the circular and quasicircular orbits. First order perturbations were determined in orbital elements taking into consideration the tidal forces and the relativistic Lense-Thirring precession. A study of nutation of the rigid Earth was performed. The theoretical study, a Hamiltonian approach in which the Andoyer variables were used, and the numerical applications pointed out the fact that Kubo's approximate value for the Chandler period (441.6 days) can be corrected ( 435.4 days) by taking into account a triaxial Earth. The possible correlation between the occurrence of earthquakes in Rumania over a very long period (984-1989) and the value of the luni-solar potential was studied. An approach was proposed, based on the Kilston-Knophoff method.

\subsection{SPACE RESEARCH CENTRE OF THE POLISH ACADEMY OF SCIENCES, WARSAW, POLAND}

The Fourier Transform Band Pass Filter (FT BPF) with the appropriate transfer functions has been discussed (Popiński \& Kosek 1995), its frequency resolution being better than that of the Wavelet Transform (Kosek 1995). The Wavelet Transform was applied to polar motion series, enabling investigation of prograde and retrograde oscillations (Popiński \& Kosek 1994). The discrete Wavelet Transform band pass filter (WT BPF) was used to compute seasonal and short-period oscillations of the polar motion data (Popiński \& Kosek 1995).

The new autocovariance prediction method was applied to improve by a factor of two the accuracy of polar motion predictions (Kosek 1993, Kosek et al. 1995). The prediction error of polar motion mostly depends on the starting prediction epochs due to irregular variations, these irregular variations were found also in amplitudes and phases of the semi-annual and 120-day oscillations (Kosek \& Kolaczek 1995). Epochs of these irregular variations coincide with El Niño events.

Time variable band pass filter spectra of polar motion with periods of 20-150 days, their excitation functions and EAAM excitation functions were computed using the Ormsby and the FT BPF (Kosek et al. 1995, Kolaczek 1995), using the IERS $90 \mathrm{C} 04$ and CSR 94L01 data. The amplitude variations (for periods shorter than 70-80 days) ranging from about 1 to 3 mas are modulated with the period of 2-3 years. The most energetic maxima of oscillations with periods longer than $70-80$ days follow the El Niño events 
in 1979, 1983-1984, 1987-1988 and about 1991. It was found that these are counterclockwise elliptic oscillations with variable amplitudes, ranging from about 2 to 5 mas (Kolaczek 1993, 1995, Kołaczek \& Kosek 1993, 1994, Kosek \& Kaczkowski 1994, Kosek et al. 1995). Correlations of the short-period oscillations of polar motion excitation functions with those of the EAAM in 1979-1995 reached maxima of 0.8 . The correlations show also annual variations achieving maxima during northern hemisphere winters (Nastula 1995, Kosek et al. 1995). Coherence between atmospheric and geodetic polar motion excitation has maxima that follow El Niño events (Kosek et al. 1995, Kolaczek 1995). Assuming that the quasi 3-year modulation of the semi-annual oscillation (Kolaczek et al. 1991) can be caused by beating of the semiannual and the semi-Chandler ones, they were filtered by the FT BPF with very narrow bandwidth using the astrometric polar motion data from 1898 to 1988 (Vondrák 1995), and the IERS 90C04 polar motion data in the period 1962-1995 (Kolaczek 1995, Kolaczek \& Kosek 1996). The semi-annual oscillation is an elliptic oscillation with its semi-major axis directed along the Greenwich meridian. Both oscillations have slightly variable amplitudes modulated with the periods of about 14 and 11 years, respectively. The variable amplitude of the semi-Chandler oscillation is weakly correlated with the 11-year solar cycle similar to the variable amplitude of Chandler nutation (Korsun et al. 1994). The same oscillations were also detected in time variable spectra of atmospheric and geodetic excitation functions.

The zonal EAAM functions in 8 latitude belts in the years 1987.6-1992.6 and in 96 latitude belts in the years 1988.3-1992.6 were computed from the Japanese Meteorological Agency data (Nastula et al.1995, Nastula 1995). 45-70 day prograde and 20-30 and 30-60 day retrograde oscillations can be seen in the spectra of EAAM equatorial components (Nastula et al. 1995). These oscillations have distinct annual modulation, especially in mid-latitude belts. Correlation coefficients, coherence and cross spectra between geodetic and zonal atmospheric excitation functions show that the EAAM functions of the Northern Hemisphere have the most important influence on excitations of polar motion with periods smaller than 150 days. Correlation coefficients between polar motion and zonal EAAM excitation functions reach their maximum values in the Northern Hemisphere, especially in the mid-latitudes (Nastula et al. 1995, Kosek et al., 1995). The equatorial components of the zonal EAAM functions show the shift between the results computed for 00h UTC and $12 \mathrm{~h}$ UTC. The distribution of these differences significantly depends on latitude. The amplitude of these semi-diurnal differences is of the order of 0.03 mas or 0.3 mas for pressure and wind terms, respectively (Nastula 1995).

Brzeziński $(1994,1996)$ studied subsequent refinements of the polar motion equation in order to meet the current observational requirements. The final result, the "broad-band" Liouville equation of polar motion (Brzeziński 1994), uses as variables only the observed quantities which are the terrestrial or the celestial coordinates of the Celestial Ephemeris Pole (CEP) and the equatorial Effective Angular Momentum (EAM) function, and remains valid for high frequency excitations including nearly diurnal retrograde variations. Brzeziński \& Capitaine (1996) discussed the definition of the CEP in view of the recent developments in the techniques monitoring Earth rotation and taking into account a permanent progress in modeling and determination of the related excitation quantities. Attention has been paid to the stochastic modeling of polar motion and its free oscillations, the Chandler wobble and the free core nutation (Brzeziński 1994c; Petrov et al. 1996). Brzeziński (1994, 1995) demonstrated new abilities of the maximum entropy method of spectral analysis, which can be particularly useful in Earth rotation investigations. The most important achievements of Brzeziński are summarized in (Zharkov et al. 1996).

\subsection{INSTITUT FÜR ANGEWANDTE GEODÄSIE, GERMANY (IFAG)}

The Fundamental Station Wettzell operated by If AG is contributing data for monitoring Earth orientation parameters by means of SLR, VLBI and GPS measurements. Investigations were carried out of the behaviour of avalanche photo diodes and two colour ranging in addition to the regular tracking of artificial satellites as Lageos $1+2$, Etalon $1+2$, Starlett, Stella, Ajisai, ERS1 + 2, Topex/Poseidon, GPS35 + 36, MeteorIII and Glonass. The Wettzell VLBI Station participated in several international programs, in particular IRIS and NEOS. The permanently tracking GPS receiver at Wettzell is one of the fixed stations in the global IGS network.

The IfAG is realizing a concept of a Transportable Integrated Geodetic Observatory (TIGO) equipped with laser ranging, VLBI, GPS, gravity, seismic, time and supplementary terrestrial measurement techniques. The installation will support the global network of Fundamental Stations which has an insufficient and unbalanced distribution especially in the southern hemisphere. 
SLR solutions for Earth orientation parameters EOP(IFAG) 96 L01 (3-d values) and station positions SSC(IFAG) 96 L01 were derived by IfAG on the basis of Lageos1 data for the year 1995 and transmitted to the IERS Central Bureau for inclusion in the annual global solutions.

\subsection{GEOFORSCHUNGSZENTRUM, POTSDAM, GERMANY}

The possible influence of global change on the rotation of the Earth was studied, using theoretical models of climate variations. It was found that mass redistributions between the cryosphere and the hydrosphere due to global warming cause effects on the Earth's rotation which are expected to be determinable after ten years; it is difficult to attribute these small effects to a particular geophysical excitation. From models of long term variations of the atmospheric dynamics it follows that the expected quantities influencing the Earth's rotation are too small to cause observable effects in variations of the length of day (LOD) and polar motion. But, from the analysis of polar motion and LOD it follows that these phenomena contain a number of periodicities which can be identified as climate cycles. Assuming that atmospheric dynamics is a possible candidate for exciting climate cycles in LOD and polar motion, the excitation functions of polar motion and LOD were calculated for the period 1900-1990. Analyses of the amplitude spectra of LOD and its atmospheric excitation function showed that the short climate cycles $(11,22$, and 35 years) are caused by atmospheric dynamics up to $50 \%$. The total excitation function of polar motion, its atmospheric excitation function, and the excitation function of water storage have similar amplitude spectra which contain all well known climate cycles, but the 11 and 22 years periods are probably caused by atmospheric dynamics only. In the amplitude variations of the Chandler wobble, climate cycles from 22 to 150 years were detected, which implies the excitation of these cycles by small variations of geophysical processes, due to the large amplification near the Chandler wobble. The climate cycles in atmospheric dynamics being too small to explain the variations of the components of the Earth's rotation, relations between climate parameters and geomagnetic field parameters were studied. A close correlation between both phenomena was found; it was suggested that climate cycles in LOD are transferred via core mantle coupling (Jochmann \& Greiner-Mai 1996).

Investigations of electromagnetic core-mantle coupling were improved using time series of LOD corrected for atmospheric effects. This correction allowed the choice of a lower electric conductivity in the lower mantle, and caused a better adaption of the amplitude spectra of mechanically and electromagnetically calculated torques (Greiner-Mai 1995).

Seasonal variations of the length of day and polar motion were studied. It was found that this period of polar motion cannot be explained completely by atmospheric excitation; the influence of another geophysical process is implied. Similar results were obtained for the seasonal variation of LOD, but, the difference between LOD and its atmospherically excited part is much smaller (Höpfner 1995).

\subsection{UNIVERSITY OF DURHAM \& ROYAL GREENWICH OBSERVATORY, CAMBRIDGE, UK}

Records of solar and lunar eclipses in the period $700 \mathrm{BC}$ to $\mathrm{AD} 1600$ originating from the ancient and medieval civilizations of Babylon, China, Europe and the Arab world were amassed and critically appraised for their usefulness in answering questions about the long-term variability of the Earth's rate of rotation. Results from previous analyses of lunar occultations in the period AD 1600-1955.5 and from high-precision data in AD 1955.5-1990 were included in the data (Stephenson \& Morrison 1995).

Using the change in the length of the mean solar day (LOD) as the measure of acceleration in the rate of rotation, it was found that the LOD has increased by $(+1.70 \pm 0.05) \mathrm{ms} / \mathrm{cy}$ on the average over the past 2700 years. Yet an increase of $+2.3 \pm 0.1 \mathrm{~ms} / \mathrm{cy}$ is expected from the tidal braking of the Earth's spin, assuming a value of $-26.0 " / \mathrm{cy}^{2}$ for the tidal acceleration of the Moon. There is thus an average accelerative component in the Earth's rotation which acts to shorten the LOD by $(-0.6 \pm 0.1) \mathrm{ms} / \mathrm{cy}$. This result is consistent with the change in LOD implied by modern measurements of $\dot{J}_{2}$ and the theory of postglacial rebound. Moreover, it was found that besides this accelerative component there is a fluctuation in the LOD with a semi-amplitude of $\sim 4 \mathrm{~ms}$ and a period of $\sim 1500 \mathrm{yr}$.

Fluctuations in the Earth's rotation since 1830, as evidenced by changes in LOD, were derived from astronomical data having sub-annual resolution by Jordi et al. (1994). Before 1955.5 timings of lunar occultations were used; after 1955.5 the data were taken from the time series TAI-UT1. Although the data in the period 1830-1890 displayed decade fluctuations in LOD, they were not accurate enough to reveal interannual variations. In this regard, also, the results from 1890-1925 were somewhat dubious. 
The quality of the data after 1925 , though, was such that the temporal behaviour of the interannual fluctuations in LOD could be traced with confidence. The interannual fluctuations in LOD since 1925 were compared with an index of the El Niño/Southern Oscillation (ENSO) phenomenon in the oceanatmosphere system and were subjected to spectral analysis. The results supported the conclusions reached by other authors that these fluctuations are linked to circulation changes in the atmosphere associated with ENSO, and in part to the quasi-biennial oscillation in the equatorial stratosphere's zonal winds. A spectral analysis of our 62 -yr series of length of day values since 1925 revealed two significant peaks in the interannual range 2-4 yr. One was roughly biennial and the other was about twice this period, broadly supporting results obtained previously from shorter records.

\subsection{STATE UNIVERSITY OF NEW YORK AT BINGHAMTON, USA}

The theoretical framework for determining the rotational response of a deformable Earth which includes fluid oceans was developed by Dickman (1993). This 'broad-band' theory accounted fully for the dynamical behavior of the oceans and included parameterizations of the solid Earth and fluid core responses valid at periods from diurnal to decadal. It was the basis for predictions of the rotational perturbations caused by long-period ocean tides, those predictions were incorporated into the 1992 standards of the IERS.

By comparing observed UT1 with theoretical predictions Dickman \& Nam (1995) were able to infer limits on the influence of mantle anelasticity and core-mantle coupling at periods between 1 and 2 weeks.

A long-standing and controversial problem in the study of Earth's rotation has been to explain the properties (enhanced amplitude and "eastward intensification") exhibited in the North Sea by the pole tide, the oceanic response to the Chandler wobble. Xie \& Dickman (1996) extend the data analyses of the North Sea tide to time spans exceeding a century, and compare the tide at North Sea ports to that predicted based on the observed polar motion during the same time spans. The authors find that the pole tide properties have been very different over different time intervals, and conclude that - although there is clearly some connection between the pole tide and meteorological processes - there must be non-rotational, non-meteorological causes of the observed pole tide characteristics as well.

\subsection{ASTRONOMICAL INSTITUTE \& THE CZECH TECHNICAL UNIVERSITY, PRAGUE, CZECH R.}

The Earth orientation parameters as determined by optical astrometry since the beginning of the century were used to determine corrections to long-periodic terms of nutation and to study long-periodic polar motion. It is shown that the largest discrepancies between optical astrometry and VLBI are found in the principal and annual nutation terms. The secular polar motion of $0.346^{\prime \prime} /$ cy toward $75.8^{\circ}$ meridian as well as the "Markowitz wobble" with a period of $29 \mathrm{yr}$ and amplitude 0.02 " are confirmed (Vondrák \& Ron 1994, Vondrák, Ron \& Čepek 1994, Vondrák \& Ron 1996).

Analysis of long term observations by the method of equal altitudes made by astrolabes and circumzenithals of different types showed the presence of systematic deformations of the apparent almucantar of the instrument (Pešek, Vondrák, Chollet \& Noël 1993, Pešek 1995). The deformations are of the order of 0.1 " -0.2 " and they vary with azimuth and time, with a dominating annual period. They are most probably caused by local refraction anomalies, (systematic) catalog errors, and also by the non-uniform distribution of stars within the observation group, while personal and instrumental influences can be excluded.

Optical positions and proper motions of seventeen radio stars were improved from observations by the method of equal altitudes as performed by circumzenithals in Prague, Pecný and Bratislava during 1980-1994 (Pešek, Hefty \& Skoupý 1996). Average accuracy of right ascensions, declinations and their proper motions is $0.004 \mathrm{~s}, 0.04 \%, 0.001 \mathrm{~s} / \mathrm{y}$ and $0.01 \% / \mathrm{y}$, respectively. 